\title{
Association between serum NSE and metabolic parameters determined by 18F-FDG PET/CT in pediatric patients with neuroblastoma
}

\author{
Shuai Man ${ }^{1}$, Jie $\mathrm{Yan}^{2}$, Jie $\mathrm{Li}^{3}$, Yan $\mathrm{Cao}^{3}$, Jianjing Liu ${ }^{2}$, and qiang zhao ${ }^{2}$ \\ ${ }^{1}$ Tianjin Medical University \\ ${ }^{2}$ Tianjin Medical University Cancer Institute and Hospital \\ ${ }^{3}$ Tianjin Tumor Hospital
}

June 1, 2020

\begin{abstract}
Purpose: To explore the correlations between neuron-specific enolase (NSE) and the metabolic parameters such as the maximum uptake (SUVmax), metabolic tumor volume (MTV) and total lesion glycolysis (TLG), determined by fluorine-18 fluorodeoxyglucose (18F-FDG) positron emission tomography-computed tomography (PET/CT) in pediatric patients with newly diagnosed neuroblastoma(NB). Method: Data from 43 patients with newly diagnosed NB between December 2013 and December 2019 were collected. The serum levels of NSE were measured at the time of diagnosis, and 18F-FDG PET/CT examinations were performed within 1 weeks. The metabolic parameters of the primary tumor lesion s such as SUVmax, MTV and TLG were calculated by 18F-FDG PET/CT. Pearson correlation analyses were applied to investigate the correlations between the serum levels of NSE and PET/CT findings. Result: NSE had strong correlations with SUVmax, MTV and TLG $(\mathrm{r}=0.521, \mathrm{P}<0.001$; $\mathrm{r}=0.520, \mathrm{P}<0.001 ; \mathrm{r}=0.442, \mathrm{P}=0.003$, respectively) using Pearson correlation analyses. The Mann-Whitney $\mathrm{U}$ tests showed that the values of SUVmax, MTV and TLG were significantly higher for the patients with NSE levels [?]100 ug/L (P=0.013, $\mathrm{P}=0.013$ and $\mathrm{P}=0.002$, respectively) and for patients with serum NSE levels larger than the cut-off value $(\mathrm{P}=0.004, \mathrm{P}=0.008$ and $\mathrm{P}<0.001$, respectively). Conclusion: In patients with newly diagnosed NB, the metabolic parameters determined by $18 \mathrm{~F}-$ FDG PET/CT could be considered as accurate markers of tumor burden, with MTV and TLG more sensitive than SUVmax. When abnormal NSE level were detected in a pediatric patient with NB, the higher the NSE level was, the larger the SUVmax MTV and TLG were.
\end{abstract}

\section{Hosted file}

$\backslash$ begin $\{\mathrm{CJK}\}\{\mathrm{UTF} 8\}\{$ gbsn\} \end } \{ \mathrm { CJK } \} \backslash \text { selectlanguage\{english\}(1).doc available at https: } //authorea.com/users/328282/articles/455659-association-between-serum-nse-and-metabolicparameters-determined-by-18f-fdg-pet-ct-in-pediatric-patients-with-neuroblastoma

\section{Hosted file}

table. doc available at https://authorea.com/users/328282/articles/455659-association-betweenserum-nse-and-metabolic-parameters-determined-by-18f-fdg-pet-ct-in-pediatric-patientswith-neuroblastoma 

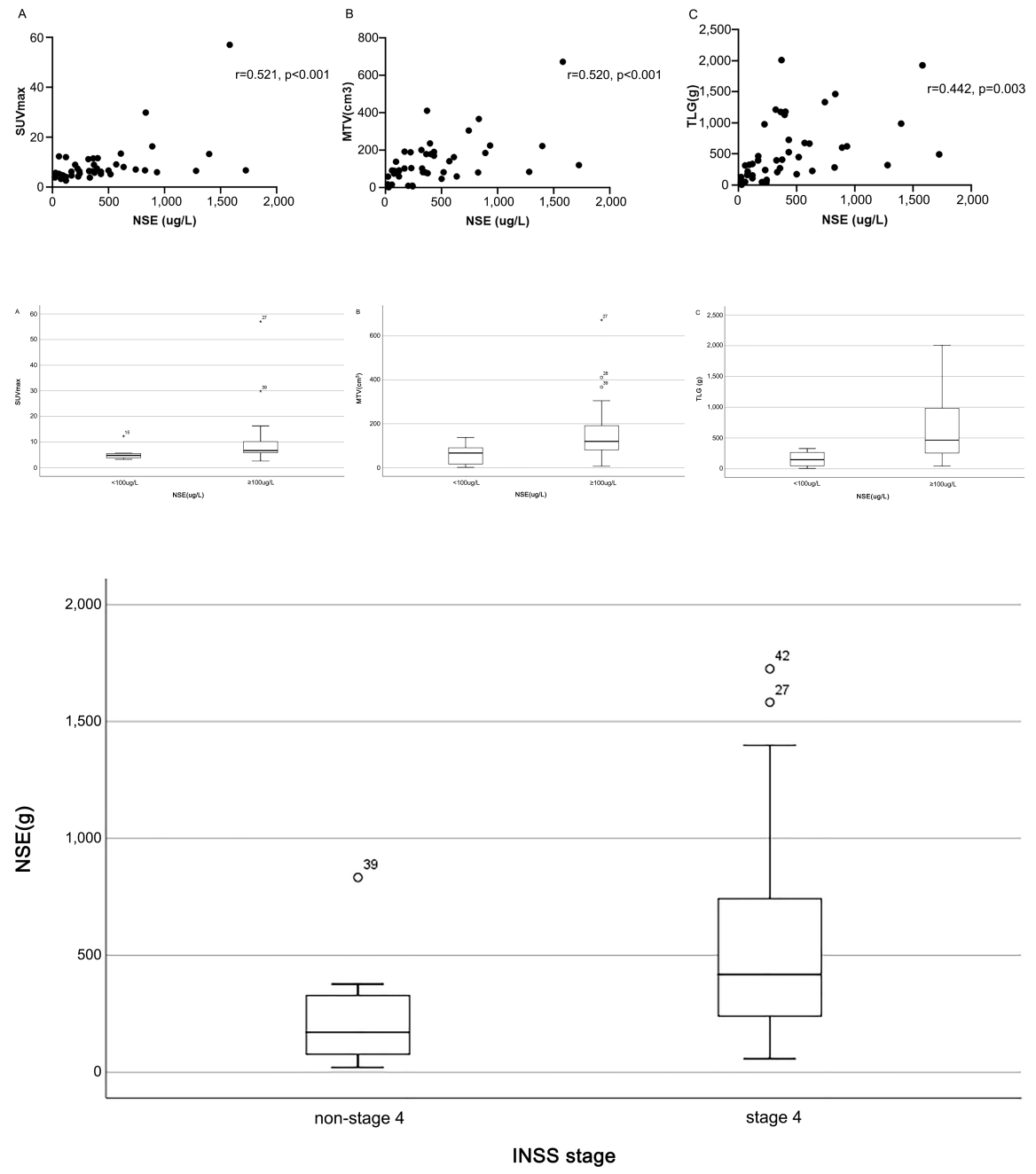

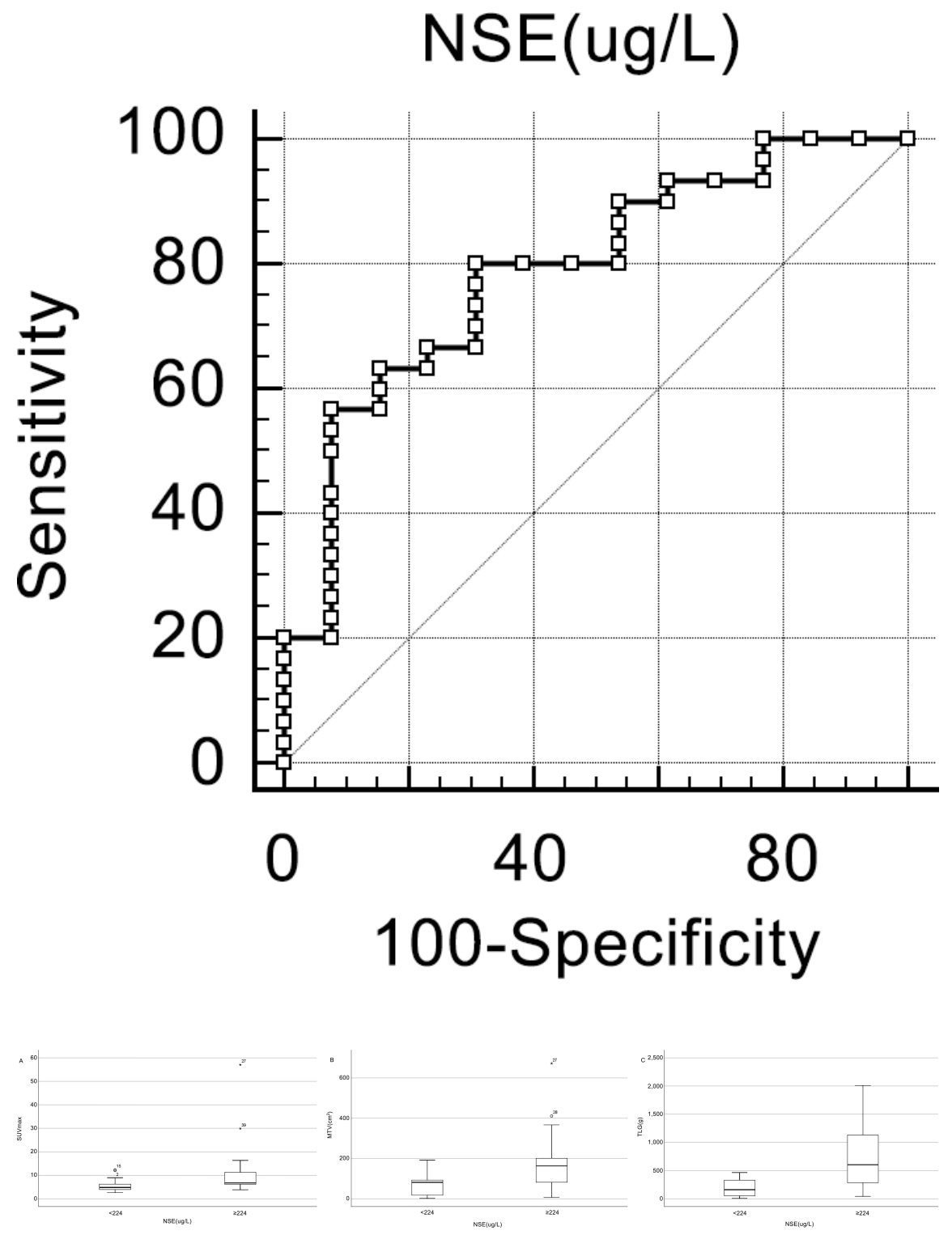